\title{
Possible challenges posed by new 2017 ACC/AHA guidelines for management of hypertension in diverse populations
}

\section{Introduction}

Although hypertension is a disease of unknown etiology, it is associated with multiple risk factors such as obesity, diabetes, alcoholism and increased consumption of salt. The exact physiological limits of blood pressures to make a diagnosis of hypertension are still undefined, despite several attempts by the American Heart Association, American College of Cardiology and International College of Cardiology. It is well accepted that out of all the noncommunicable disease (NCD), high blood pressure is second most common problem after obesity. ${ }^{1,2}$ The Five City Study in India, revealed that hypertension was commonest in South and West India compared to North and east India. ${ }^{2}$ Of all the above risk factors of hypertension, obesity, physical inactivity appear to be most common, followed by increased intake of salt, alcoholism and family history in most of the studies. ${ }^{2}$

The normal physiological limits of many clinical and behavioral risk factors such as body weight, blood pressures, waist/hip ratio, moderate alcohol intake, moderate exercise training as well as prayer and meditation are not known. Therefore, the exact physioligical limits of these marker need further research.

It is well known that the magnitude of reductions in total and cardiovascular mortality can be increased if other risk factors are also decreased along with decline in blood pressure among patients with hypertension..$^{1-3}$ The risk of mortality after low-density lipoprotein (LDL) cholesterol lowering depend on the baseline LDL-cholesterol level as well as on presence of other risk factors such as hypertension and type 2 diabetes mellitus. ${ }^{3}$ There is some evidence that it is oxidized cholesterol which is atherogenic, whereas native cholesterol may not be absolutely unhealthy. However, a recent meta-analysis involving 34 randomized clinical trials, including 270288 subjects, revealed that more intensive lowering of LDL cholesterol by statin therapy was associated with a marked decline in total mortality with higher baseline concentrations of LDL cholesterol (rate ratio, 0.91 for each $40-\mathrm{mg} / \mathrm{dL}$ increase in baseline level). The relationship was absent when the baseline LDL-C level was less than $100 \mathrm{mg} / \mathrm{dL}$. Cardiovascular mortality also revealed a similar relationship which means that the greatest benefit from reduction of LDL cholesterol can occur for patients with baseline LDL cholesterol concentration of 100 $\mathrm{mg} / \mathrm{dL}$ or greater. ${ }^{3}$

The global burden of hypertension and systolic blood pressure [SBP] which were 110 to $115 \mathrm{~mm} \mathrm{Hg}$, were reported for the years 1990 to 2015 in the GBD study. ${ }^{4}$ Further analysis revealed that SBP of at least $110 \mathrm{~mm} \mathrm{Hg}$ could be a strong predictor of chronic kidney outcomes as well as coronary artery disease [CAD], stroke, and heart failure. ${ }^{4}$ Despite our understanding about the role of diet in hypertension, the burden of systolic BP of $110 \mathrm{mmHg}$ is considered high, which should be treated by drug therapy ${ }^{5-8}$ Since obesity is the commonest risk factor of hypertension, the global obesity epidemic

\author{
Volume 7 Issue 3 - 2018 \\ RB Singh,' Sergey Chibisov, ${ }^{2}$ Omar Al- \\ bawareed tha, ${ }^{2}$ Maria Abramova, ${ }^{2}$ Mikhael L \\ Blagonravov, ${ }^{2}$ Anuj Maheshwari, ${ }^{3}$ Narsingh \\ Verma, ${ }^{4}$ ViliamMojto ${ }^{5}$ \\ 'Halberg Hospital and Research Institute, India \\ ${ }^{2}$ People,s Friendship University of Russia, Russia \\ ${ }^{3}$ King George's Medical University, India \\ ${ }^{4}$ Babu Banarasi Das University, India \\ ${ }^{5}$ Comenium University, Slovakia
}

Correspondence: Sergey Shastun,MD, Division of Chronomedicine, People,s Friendship University of Russia, Moscow, Russia, Tel +792647/2428,

Email kalcna@mail.ru

Ram B Singh, Halberg Hospital and Research Institute, Civil Lines, Moradabad, India, Tel +91 9997794 I02,

Email rbs@tsimtsoum.net

Received: March 24, 2018 | Published: May 24, 2018

or a modest increase in body mass index above $23 \mathrm{Kg} / \mathrm{m}^{2}$ may cause central obesity and further increase systolic BP in some populations such as South Asians. ${ }^{2}$ There were 8.69 million subjects, from 154 countries. It is assessed that, the rate of systolic BP of at least 110 to $115 \mathrm{~mm} \mathrm{Hg}$, has increased from 73119 to 81373 per 100000 subjects. Systolic BP of $140 \mathrm{~mm} \mathrm{Hg}$ or greater increased from 17307 to 20526 per 100000 subjects. The rate of annual deaths in association with systolic BP of at least 110 to $115 \mathrm{~mm} \mathrm{Hg}$ is higher from 135.6 to 145.2 per 100000 subjects, and for systolic BP of $140 \mathrm{~mm} \mathrm{Hg}$ or higher increased from 97.9 to 106.3 per 100000 subjects according to new estimates. ${ }^{4}$ Therefore, it appears that during the last 25 years, there is worldwide marked increase in estimated deaths with systolic BP levels of at least 110 to $115 \mathrm{~mm} \mathrm{Hg}$ and of $140 \mathrm{~mm} \mathrm{Hg}$ or higher. According to European guidelines, it is possible to stratify all the patients with hypertension into four categories (Table 1).

Table I Categories of high blood pressure as per earlier guidelines High-normal blood pressure (130-139 systolic or 85-89 $\mathrm{mmHg}$ diastolic),

Grade 1 hypertension (140-159 systolic or 90-99 diastolic mmHg),

Grade 2 hypertension (160-179 systolic or 100-109 mmHg diastolic),

Grade 3 hypertension ( $>180$ systolic or $>110 \mathrm{mmHg}$ diastolic).

Hypertension has been the focus of multiple guidelines for clinical management beginning from the first Joint National Committee in 1977. In 2014, the ICC-ICN Expert Group published Sofia declaration for prevention of CVDs and type 2 diabetes mellitus as a scientific statement of the International College of Cardiology and International College of Nutrition. ${ }^{1}$ In the same year, the National Heart, Lung, 
and Blood Institute focused on a few key treatment questions, commissioned a writing group, which used only data from randomized clinical trials (RCTs) to inform their recommendations. ${ }^{9}$ All these agencies gave due consideration to management of high blood pressure, because hypertension is a modifiable risk factor of CVDs. Some of the treatment goals for subgroups were relaxed by the writing group as there was little evidence of benefit. These recommendations were; relaxation in treatment of patients aged 60 years or older and subjects with kidney disease or diabetes who are at increased risk of further complications. ${ }^{9-11}$ These conservative guidelines were criticized and finally rejected by major professional societies as well as by other writing group who wrote original guidelines. ${ }^{11}$

\section{Possible challenges posed by the new 20I7 ACCIAHA consensus guideline on high blood pressure}

There is an addition of interesting new findings from recent randomized, controlled trials, with focus on high blood pressure, the American College of Cardiology and the American Heart Association (ACC/AHA) have now produced the 2017 Guideline for the Prevention, Detection, Evaluation, and Management of High Blood Pressure in Adults. ${ }^{12-15}$ There is a summary; Clinical Guidelines Synopsis published recently in JAMA. ${ }^{14}$ Compared to 2014 guideline, the scope of this guideline appears to be quite extensive, due to the inclusion of multiple aspects such as diagnostic workup and evaluation, definition and limits of high BPs, strategies for lifestyle management for prevention and for treatment. The thresholds for BP treatment and initial pharmacotherapy, and about methods; home $\mathrm{BP}$ or ambulatory BP measurement in the long-term monitoring are also part of the guidelines. ${ }^{14} \mathrm{New}$ recommendations include many substantial changes on newer aspects of blood pressure variations in the management of high BP. ${ }^{14}$ The majority of the recommendations give is a consistent advice for a more aggressive approach for early diagnosis and treatment, consistent with growing evidence from epidemiological studies and drug trials. The interesting points are how health professionals will translate these guidelines into clinical management of high BP. The purpose is to more effectively treat high BP at population as well as individual levels because the new treatment limits are further lowered.

In some patients, other cardiovascular risk factors as well as end organ damage/disease that may be present, have to be duly addressed in the decisions for treatment of hypertension for which a full riskassessment algorithm is included in the guidelines. Diet and lifestyle advice for reducing BPs are as important as drug therapy.,16-18 Recommending salt intake of approximately 5-6 g per day, in contrast to a typical intake of 9-12g per day, may be useful to some patients, ${ }^{10}$ although the BP response to salt restriction should be assessed on an individualized basis, since in some patients, salt reduction can actually increase blood pressure, as shown by Franz Halberg as well as others. ${ }^{19-23}$ Conventional (non-chronobiologic) studies indicate that, on average, a decrease in salt intake to $5 \mathrm{~g}$ per day can cause reduction in systolic BP of about $1-2 \mathrm{mmHg}$ in subjects with normotension and 4-5 mmHg in patients with high BP. Since the optimal body mass index (BMI) is not known, it is recommended to reduce BMIs below $25 \mathrm{~kg} / \mathrm{m}^{2}$. Reduction of waist circumferences to less than $102 \mathrm{~cm}$ in men and $88 \mathrm{~cm}$ in women in Caucasians may be fine but for Asians, it is too much, who need advice for lower levels. A weight loss of about
$5 \mathrm{~kg}$ may reduce $4 \mathrm{~mm} \mathrm{Hg}$ of systolic $\mathrm{BP}$, aerobic endurance physical training can reduce systolic BP by $7 \mathrm{mmHg}$, independently of any decrease in body weight. ${ }^{10,16-18}$ Exercise also benefits brain function by increasing brain derived neurotrophic factor and neuronal growth factor. Exercise training particularly in the morning also has beneficial effects on HRV, VEGF and HDL, which protect against atherosclerosis and neuronal degeneration. ${ }^{17}$ The major recommendations of Guideline title 2017 ACC/AHA/AAPA/ABC/ACPM/AGS/APHA/ASH/ASPC/ NMA/PCNA Guideline for the Prevention, Detection, Evaluation, and Management of High Blood Pressure in Adults are given below ${ }^{12,13}$ which have also been critically evaluated by other experts..$^{14,15}$ In the diagnosis of hypertension, BPs should be categorized as given in Table 2. Other recommendations include out-of-office BP measurements for confirmation of the diagnosis of high BP as well as for change in drug therapy for reducing BP in conjunction with telehealth counseling or clinical interventions. ${ }^{12,13}$

Table 2 Categories of high blood pressures according to new guidelines

Normal $(<120 / 80 \mathrm{~mm} \mathrm{Hg})$, High (120-129/>80 mm Hg),

Stage 1 hypertension (130-139/80-89 $\mathrm{mm} \mathrm{Hg}$ ), or

Stage 2 hypertension ( $\geq 140 / 90 \mathrm{~mm} \mathrm{Hg}$ ) (strong recommendation; moderatequality evidence).

\section{Management of high blood pressure}

Initiating therapy should be initiated with non-drug interventions that are effective in lowering BP are recommended for people with elevated BP or hypertension because it has high quality evidence hence there is strong recommendation. Hristova et al. ${ }^{1}$ advise to take $400 \mathrm{~g} /$ day of fruits $(200 \mathrm{~g} /$ day) and vegetables $(150 \mathrm{~g} /$ day) and nuts (20$50 \mathrm{~g} /$ day) and another $400 \mathrm{~g} /$ day of whole grains, in conjunction with $30-100 \mathrm{~g}$ vegetable oil, preferably a blend of oils containing olive oil $(50 \%)+$ rape seed oil $(30 \%)+$ rice bran oil $(11 \%)+$ flax seed oil $(9 \%)$ to achieve adequate monounsaturated fatty acids, omega- 3 fatty acids and polyphenolics as well as other antioxidants. ${ }^{1}$ Patients with clinical CVD as well as those with an estimated 10-year atherosclerotic CVD (ASCVD) risk of $10 \%$ or higher need drug therapy. A systolic BP of $130 \mathrm{~mm} \mathrm{Hg}$ or higher or a diastolic BP of $80 \mathrm{~mm} \mathrm{Hg}$ or greater should be treated by drug therapy with expert opinion for diastolic BP. Those patients without history of CVD and an ASCVD risk of less than 10\%, drug therapy for reducing BP is advised. However, those patients having systolic BP of $140 \mathrm{~mm} \mathrm{Hg}$ or greater or a diastolic BP of $90 \mathrm{~mm} \mathrm{Hg}$ or greater, there is a strong recommendation for drug therapy although it has low-quality evidence. A target BP of less than $130 / 80 \mathrm{~mm} \mathrm{Hg}$ is advised strongly, for patients with CVD or ASCVD event risk of $10 \%$ or higher, although the quality of evidence is moderate for systolic BP. There may be a need for expert opinion on drug therapy for diastolic BP. In low-risk subjects, there is weak recommendation of moderatequality evidence for BP target of $130 / 80 \mathrm{~mm} \mathrm{Hg}$. Expert opinion may be desirable for drug therapy for diastolic BP. Pharmacotherapy as first line should include; thiazide diuretics, calcium channel blockers (CCBs), and angiotensin-converting enzyme (ACE) inhibitors or angiotensin II receptor blockers (ARBs) with strong recommendation and high-quality evidence. Patients can begin treatment with 2 first line drugs of different groups, in stage 2 hypertension and an average BP of more than 20/10mm Hg above. 


\section{The clinical problem and challenges of high blood pressure}

Hypertension is a leading risk factor for mortality and disability and it is estimated that 874 million adults worldwide have an SBP of $140 \mathrm{~mm} \mathrm{Hg}$ or higher. ${ }^{12,13}$ Hypertension is second only to cigarette smoking, as a preventable cause of death with its association of CVDs; stroke (cerebrovascular accident [CVA), heart failure, and chronic kidney disease (CKD). ${ }^{16}$ The complications are likely to increase due to given demographic trends and the increasing prevalence of hypertension with increasing age; $79 \%$ of men and $85 \%$ of women $>75$ years old have hypertension. ${ }^{12,13}$ This guideline was developed by; The ACC/AHA Task Force on Clinical Practice Guidelines which selected a writing committee that was notable for its inclusion of people with a breadth of backgrounds, wide scope of practice, and freedom from conflicts of interest. The guideline provides a comprehensive overview of the diagnosis and therapy of hypertension with 106 graded recommendations divided into 47 modular knowledge chunks with 8 recommendations of clinical covered in this JAMA Clinical Guidelines Synopsis. This guideline recommends classifying BP into 4 categories as given in Table 2. These categories of BP are made for facilitation of making decisions for clinical and public health objectives. They also indicate data on observation for suggesting an extent or severity of risk of CVD as BP increases from normal to elevated to hypertension stages 1 and $2 .^{12,13}$

This guideline recommends routine use of out-of-office BP measurements (home or ambulatory BP monitoring) in the diagnosis and treatment of hypertension, although it is known that 24hour ambulatory BP is an independent risk factor for future CVDs. This recommendation reflects the differences between office and home BP values with focus on masked hypertension and white-coat hypertension and acknowledges that the tighter BP goals recommended in this guideline may require closer BP monitoring. The evidence for this recommendation was reviewed in one of the commissioned systematic reviews. ${ }^{17}$ Compared with usual care, patients using home $\mathrm{BP}$ monitoring had a greater reduction in office $\mathrm{S}$ systolic BP at 6 months vs office-measured BP (4.9 [95\% CI, 1.3-8.6] mm Hg). This result did not persist at 12 months. There was also no evidence of clinical benefit. Non-pharmacologic interventions are strongly supported in the guideline for their primary and complementary effect in lowering BP. These interventions include weight loss in patients who are overweight or obese; a heart-healthy diet, such as the DASH (Dietary Approaches to Stop Hypertension) diet; sodium reduction; potassium supplementation; increased physical activity; and moderation of alcohol consumption. ${ }^{18}$ Most of these interventions have been shown in randomized trials to reduce systolic BP by 5 to $10 \mathrm{~mm} \mathrm{Hg}$. Weight loss has been shown to decrease BP by about $1 \mathrm{~mm}$ $\mathrm{Hg}$ per $1 \mathrm{~kg}$ of weight loss. ${ }^{12,13}$ Taking of the DASH diet yielded an 11-mm Hg decrease in SBP. ${ }^{18}$

Drug therapy is recommended for patients with or at high risk of CVD at BP levels of $130 / 80 \mathrm{~mm} \mathrm{Hg}$ or higher. Drug therapy is also recommended for patients without and at low risk of CVD at 140/90 $\mathrm{mm} \mathrm{Hg}$ or higher. The guideline recommends using an ASCVD risk score indicating the estimated 10-year risk of myocardial infarction, CVA, or CAD death, of $10 \%$. In other recent hypertension guidelines, such risk stratification has been used which could help translate grouplevel evidence from trials to individual patients. ${ }^{19}$ For individuals with or at high risk of CVD, the lower systolic threshold is well supported by data from a patient-level meta-analysis. ${ }^{20}$ Drug therapy in patients without and at low risk of CVD at BPs $\geq 140 / 90$ is unchanged from the JNC 7 and JNC 8 recommendations because the evidence for this recommendation is strong but mostly indirect. A meta-analysis of patients without CVD and with BP levels of $140 / 90$ to $159 / 99 \mathrm{~mm}$ $\mathrm{Hg}$ who were randomly assigned to an antihypertensive vs a control (placebo in 95\%; less intensive regimen in 5\%) BP-lowering regimen show interesting findings. ${ }^{21}$ The odds ratios for events over the 5 years in this study were 0.72 (95\% CI, 0.55-0.94) for stroke, $0.75(95 \%$ CI, 0.57-0.98) for cardiovascular death, and 0.78 (95\% CI, 0.67$0.92)$ for overall mortality. The BP difference in this study between treatment and control groups was only $3.6 / 2.4 \mathrm{~mm} \mathrm{Hg}$ and $96 \%$ of the patients had diabetes mellitus and the benefits in CVDs were only non-significant.

In another systematic review commissioned for this guideline, ${ }^{17}$ included trials that compared an systolic BP target of less than $130 \mathrm{~mm} \mathrm{Hg}$ with any higher target, patients benefited in terms of major cardiovascular events (relative risk [RR], 0.84; 95\% CI, 0.73 0.99 ) and stroke (RR, 0.82; 95\% CI, 0.70-0.96) but not myocardial infarction (RR, 0.85 ; 95\% CI, 0.73-1.00) or all-cause mortality (RR, 0.92 ; $95 \%$ CI, 0.79-1.06). Similar to the data on initiating therapy, very little information from clinical trials is available to guide recommendations about treating DBP and low risk patients. The choice of initial pharmacologic therapy for hypertension were based on another commissioned systematic review. ${ }^{17}$ This meta-analysis examined trials that compared any 2 of the antihypertensive classes: thiazides, ACEIs, ARBs, CCBs, and $\beta$-blockers. A total of 152379 patients were included in this meta-analysis, with an average of 3.5 years of follow-up and the results showed that in terms of allcause mortality, all classes were similar. Thiazides showed added benefits on secondary end points; CVA, cardiovascular events, and heart failure. Recommendation was for thiazide diuretics, CCBs, ACE inhibitors, or ARBs as first-line agents depending upon comorbidities; $\mathrm{CAD}$, heart failure, $\mathrm{CKD}$, acute stroke, and hypertensive emergency, among others. The use of 2 first-line agents of different classes for patients with stage 2 hypertension remains unchanged since JNC 7. These therapies are based on expert opinion based on studies using fixed-dose combinations that show greater BP lowering and better adherence to therapy with fixed-dose combinations. ${ }^{12,13}$

The JNC 7 categorized stage 1 hypertension as a BP of 140$159 / 90-99 \mathrm{~mm} \mathrm{Hg}$ and recommended $140 / 90 \mathrm{~mm} \mathrm{Hg}$ as the threshold for initiation of antihypertensive drug therapy in the general adult population and $130 / 80 \mathrm{~mm} \mathrm{Hg}$ or higher for patients with diabetes or CKD. The JNC 8, published in 2014, recommended that for people aged 60 years or older, pharmacologic therapy should be initiated at a BP of $150 / 90 \mathrm{~mm} \mathrm{Hg}$ or higher and treated to a goal of less than that number. In the ACCORD trial, there was an increase in serious adverse events attributable to BP medications $(3.3 \%$ vs $1.27 \% ; P<.001) .{ }^{22}$ In the SPRINT trial, there were increases in hypotension $(2.4 \%$ vs $1.4 \% ; P<.001)$ and syncope $(2.3 \%$ vs $1.7 \% ; P=.05)$, despite no overall increase in adverse events. ${ }^{23}$ The potential harms associated with the adoption of this guideline are the adverse effects of medications and tight BP and the costs of overuse of medication and home BP monitors, if the treatment thresholds and targets are shown to be overly aggressive. In view of the high attributable risk of heart disease and stroke due to high BP, from the public health point of view, the benefits of tighter control of BP appear to be greater. Randomized trials as well as observational and modeling studies support the idea that lower treatment thresholds and targets are beneficial in higher-risk patients, since progressively greater absolute risk reductions occur as 
baseline risk increases. ${ }^{17,24}$ Many studies indicate that lowest levels in BP can improve outcome in most patients, including elderly above 75 years. Therefore, the balance of the cost of medication, adverse effects and potential benefits of BP control as well as polypharmacy should be evaluated for each subject. Shared decision making between patients and their clinicians is required to arrive at an optimal treatment plan for each patient. There is little high-quality evidence in the literature about some patient populations; most notably the frail elderly who also need similar targets of BPs. ${ }^{24}$ There is a need to have team based approach with, electronic medical record, and population health approaches to BP control.

However, these guidelines are not final because they are based on the findings that one treatment is better than other, without an absolute risk reduction. Home BP, self-measurements as a supplement to clinic $\mathrm{BP}$ are unable to quantify the night time/asleep BP and pattern of 24- $h$ BP levels, hence there could be wrong classification up to $50 \%$ of all evaluated subjects. In subjects who are likely to have a blunted dipping or nighttime $\mathrm{BP}$ decline, ABPM should be a priority because there may be an increased risk of CVD. These subjects are elderly, may have obesity or overweight and secondary or resistant hypertension, and those diagnosed with CKD, sleep disorders, metabolic syndrome, and type 2 diabetes. ABPM could be a new gold standard for assessment of accurate risk due to high $\mathrm{BP}$ and diagnose true hypertension as well as consequent tissue/organ, maternal/fetal, and CVD risk, for suitable therapy of hypertension. ${ }^{25-27}$

Joint recommendations from various international agencies such as the International Society for Chronobiology (ISC), American Association of Medical Chronobiology and Chronotherapeutics (AAMCC), Spanish Society of Applied Chronobiology, Chronotherapy, and Vascular Risk (SECAC), Spanish Society of Atherosclerosis (SEA), and Romanian Society of Internal Medicine (RSIM) also support the idea that $24 \mathrm{~h} \mathrm{BP}$ assessment by ABPM is gold standard in the assessment of risk of high BPs. ${ }^{25}$ However, there is a need to design drug trials with adequate number of patients with adequate power for clinical end points. Since lower BP is associated with better outcomes, future trials should refine knowledge regarding the balance between harms and benefits of BP treatment, particularly among patients with stage 1 hypertension by assessment of risk via 24 hour ABPM. ${ }^{28}$

Some experts believe that it is more worrisome because many experts are critical at the new guidelines for making too many individuals in the United States "sick" or labeling them with a disease ${ }^{29}$ After the cholesterol guideline appeared, similar opinions were expressed that the "new guideline" turned billions of people into candidates for statins. ${ }^{30}$ Now, drug industry would be delighted, because too many people will be defined as hypertensive because of a new lower threshold for diagnosis and treatment of hypertension. Therefore, ABPM appears to be gold standard because many subjects diagnosed to be hypertensive by following new guidelines, may be actually normotensives on assessment with ABPM. ${ }^{28,31}$ Indian Society of Chronomedicine and Lebnon Society of Chronomedicine as well as Russian Society of Chronobiology and Chronomedicine emphasize on taking 7-day ABPM for the diagnosis of hypertension before any drug therapy is given to these patients. ${ }^{31}$ Previous research has demonstrated that magnetic forces and storms in the space can influence biorhythms and blood pressures which need to be given due consideration while drugs are administered for reducing blood pressures. ${ }^{32-35}$ People taking alcohol regularly also need special attention in measuring BPs and administering drug therapy because therapy varies according to alcohol intake. ${ }^{36}$

In brief, multifaceted interventions in few trials, that include outof-office BP measurements are critical to understand and it is not clear that these measurements considered in the decisions for management will prove truly beneficial. The assessment of threshold for BP treatment to individual cardiovascular risk is attractive, which should be assessed by ABPM by a $24 \mathrm{~h}$ BP record, preferably 7 day record, taken half hourly, because it could concentrate efforts on patients who will obtain the most benefit. It is also possible that the greater complexity of applying guidelines based on ASCVD risk could undermine the expected benefit. Support with electronic health record which calculates and trends individual ASCVD risk, may reduce the burden in clinical practice but needs further research.

\section{Acknowledgements}

International College of Cardiology for logistic support.

\section{Conflict of interest}

Conflict of interest has not been declared by the authors.

\section{References}

1. Hristova K, Pella D, Singh RB, et al. Sofia declaration for prevention of cardiovascular diseases and type 2 diabetes mellitus: a scientific statement of the International College of Cardiology and International College of Nutrition; ICC-ICN Expert Group. World Heart J. 2014;6(2):89-106.

2. Singh RB, Fedacko J, Pella D, et al. Prevalence and risk factors of pre-hypertension and hypertension in five Indian cities. Acta Cardiol. 2011;66(1):29-37.

3. Navarese EP, Robinson JG, Kowalewski M, et al. Association between baseline LDL-C level and total and cardiovascular mortality after LDL-C lowering: A systematic review and meta-analysis. JAMA. 2018;319(15):1566-1579.

4. Forouzanfar MH, Liu P, Roth GA, et al. Global burden of hypertension and systolic blood pressure of at least 110 to $115 \mathrm{~mm} \mathrm{Hg}, 1990$ 2015. JAMA. 2017;317(2):165-182.

5. Shastun S, Abramova M, Singh RB, et al. Diet and hypertension. World Heart J. 2014;6(4):233-236.

6. Singh RB, Rastogi SS, Singh R, et al. Effects of guava intake on serum total and high density lipoprotein cholesterol levels and systemic blood pressures. Amer J Cardiol. 1992;70(15):1287-1291.

7. Elliott P, Stamler J, Dyer AR, et al. Association between protein intake and blood Pressure; The INTERMAP Study. Arch Intern Med. 2006;166(1):79-87.

8. Yokoyama Y, Nishimura K, Barnard ND, et al. Vegetarian diets and blood pressure: a meta-analysis. JAMA Intern Med. 2014; 174(4):577587.

9. Forouzanfar MH, Liu P, Roth GA, et al. Global burden of hypertension and systolic blood pressure of at least 110 to $115 \mathrm{~mm} \mathrm{Hg}, 1990-2015$. JAMA. 2017;317(2):165-182.

10. James PA, Oparil S, Carter BL, et al. 2014 evidence-based guideline for the management of high blood pressure in adults. JAMA. 2014;311(5):507-520. 
11. Wright JT, Fine LJ, Lackland DT, et al. Evidence supporting a systolic blood pressure goal of less than $150 \mathrm{~mm} \mathrm{Hg}$ in patients aged 60 years or older. Ann Intern Med. 2014;160(7):499-503.

12. Whelton PK, Carey RM, Aronow WS, et al. 2017 ACC/AHA/AAPA/ $\mathrm{ABC} / \mathrm{ACPM} / \mathrm{AGS} / \mathrm{APhA} / \mathrm{ASH} / \mathrm{ASPC} / \mathrm{NMA} / \mathrm{PCNA}$ guideline for the prevention, detection, evaluation, and management of high blood pressure in adults: a report of the American College of Cardiology/ American Heart Association Task Force on Clinical Practice Guidelines. Hypertension.

13. Whelton PK, Carey RM, Aronow WS, et al. 2017 ACC/AHA/AAPA/ $\mathrm{ABC} / \mathrm{ACPM} / \mathrm{AGS} / \mathrm{APhA} / \mathrm{ASH} / \mathrm{ASPC} / \mathrm{NMA} / \mathrm{PCNA}$ guideline for the prevention, detection, evaluation, and management of high blood pressure in adults: a report of the American College of Cardiology/ American Heart Association Task Force on Clinical Practice Guidelines. J Am Coll Cardiol. 2018;71(19):e127-e248.

14. Greenland P, Peterson E. The New 2017 ACC/AHA Guidelines "Up the Pressure" on Diagnosis and Treatment of Hypertension. JAMA. 2017;318(21):2083-2084.

15. Cifu AS, Davis AM. Prevention, Detection, Evaluation, and Management of High Blood Pressure in Adults. JAMA. 2017;318(21):2132-2134.

16. Danaei G, Ding EL, Mozaffarian D, et al. The preventable causes of death in the United States. PLoS Med. 2009;6(4):e1000058.

17. Reboussin DM, Allen NB, Griswold ME, et al. Systematic review for the 2017 ACC/AHA/AAPA/ABC/ACPM/AGS/APhA/ASH/ASPC/ NMA/PCNA guideline for the prevention, detection, evaluation, and management of high blood pressure in adults. Hypertension. 2017.

18. Sacks FM, Svetkey LP, Vollmer WM, et al. Effects on blood pressure of reduced dietary sodium and the Dietary Approaches to Stop Hypertension (DASH) diet. N Engl J Med. 2001;344(1):3-10.

19. van der Leeuw J, Ridker PM, van der Graaf Y, et al. Personalized cardiovascular disease prevention by applying individualized prediction of treatment effects. Eur Heart J. 2014;35(13):837-843.

20. Sundström J, Arima H, Woodward M. Blood pressure-lowering treatment based on cardiovascular risk. Lancet. 2014;384(9943):591598.

21. Sundström J, Arima H, Jackson R, et al. Effects of blood pressure reduction in mild hypertension. Ann Intern Med. 2015;162(3):184-191.

22. Cushman WC, Evans GW, Byington RP, et al. Effects of intensive blood-pressure control in type 2 diabetes mellitus. $N$ Engl $\mathrm{J} \mathrm{Med}$. 2010;362(17):1575-1585.

23. Wright JT, Williamson JD, Whelton PK, et al. A randomized trial of intensive vs standard blood-pressure control. $N$ Engl $\mathrm{J} \mathrm{Med}$. 2015;373(22):2103-2116.

24. Chobanian AV. Hypertension in 2017-what is the right target? JAMA 2017;317(6):579-580.
25. Hermida RC, Smolensky MH, Ayala DE, et al. 2013 ambulatory blood pressure monitoring recommendations for the diagnosis of adult hypertension, assessment of cardiovascular and other hypertension-associated risk, and attainment of therapeutic goals. Joint recommendations from the International Society for Chronobiology (ISC), American Association of Medical Chronobiology and Chronotherapeutics (AAMCC), Spanish Society of Applied Chronobiology, Chronotherapy, and Vascular Risk (SECAC), Spanish Society of Atherosclerosis (SEA), and Romanian Society of Internal Medicine (RSIM). Clin Investig Arterioscler. 25(2):74-82.

26. Krakoff LR. Blood pressure out of the office: Its time has finally come. Amer Jour Hyperten. 2016;29(3):289-295.

27. Head GA, McGrath BP, Mihailidou AS, et al. Ambulatory blood pressure monitoring in Australia: 2011 consensus position statement. $J$ Hypertens. 2012;30(2): 253-266.

28. Singh RB, Verma NS, Hristova K, et al. Pittfalls in the 2017 ACC/AHA consensus blood pressure guidelines and the role of 24-hour ambulatory blood pressure monitoring. World Heart J. 2017;9(4):260-265.

29. Greenland P. Cardiovascular Guideline Skepticism vs Lifestyle realism. JAMA. 319(2):117-118

30. Ioannidis JP. More than a billion people taking statins? potential implications of the new cardiovascular guidelines. JAMA. 2014;311(5):463-464.

31. Singh RB, Verma NS, Maheshwari A, et al. Guidelines of the Indian Society of Chronomedicine Provide A Balanced View For ACC/AHA and International Society of Chronobiology Controversies. A View Point. World heart J. 2018;10.

32. Chibisov SM, Breus TK, Levitin AE, et al. Biological effects of aglobal magnetic storm. Biofizika. 1995;40:959-968.

33. Chibisov SM, Katinas GS, Ragulskaya MV. Biorhythms and space: monitoring relations between space and biosphere. Moscow: Publishing house "Capital Print. 2013;442.

34. Cornelissen G, Halberg F, Breus TK, et al. Nonphotic solar associations of heart rate variability and myocardial infarction. Journal of Atmospheric and Solar-Terrestrial Physics. 2002;64(5-6):707-728.

35. Singh RB, Maria Abramova, Sergey Chibisov, et al. Can gravitational waves predispose neuro-cardiovascular circadian rhythms? Clin Res Trials. 2016;2:258-259.

36. Wood AM, Kaptoge S, Butterworth AS, et al. Risk thresholds for alcohol consumption: combined analysis of individual-participan data for 599912 current drinkers in 83 prospective studies. Lancet. 2018;391(10129):1513-1523. 